\title{
Vorhersage der Schizophrenie mittels bildgebender Verfahren
}

\author{
Für die bisher ausschließlich auf der Klinik basierenden Diagnose der Schizophrenie besteht ein \\ hoher Bedarf an biologischen Markern, die bereits im Prodromalstadium einen späteren Ausbruch \\ vorhersagen können. MRT-Daten könnten diese Aufgabe übernehmen.
}

Dem Vollbild der Schizophrenie geht in der Regel eine mehrjährige Prodromalphase voraus, die durch zunehmende funktionelle Defizite und erste, überwiegend unspezifische Symptome charakterisiert ist. Der weitere Verlauf der Erkrankung könnte - eine frühzeitige Diagnose und therapeutische Intervention im Vor- oder Frühstadium vorausgesetzt - abgebremst werden. Doch in der klinischen Praxis vergehen nach wie vor drei bis fünf Jahre, bis die Diagnose einer schizophrenen Psychose getroffen wird. Denn bisher basiert die Diagnose der Schizophrenie ausschließlich auf der Klinik. Somit bestehe ein hoher Bedarf an biologischen Markern, mit deren Hilfe sich bereits im Prodromalstadium ein späterer Ausbruch mit hoher Sicherheit vorhersagen lässt. Wie Dr. Nikolaos Koutsouleris, München, berichtete, ist es mithilfe der zerebralen Magnetresonanztomografie (MRT) schon heute möglich, erste subtile neuroanatomische Veränderungen im Prodromalstadium zu detektieren, die mit einem späteren Erkrankungsbeginn korrelieren.

Im Rahmen einer großen Längsschnittstudie über 15 Jahre analysierten Koutsouleris et al. die MRT-Daten von Patienten mit Schizophrenie sowie von Patienten mit Depression und verglichen sie mit denen von gesunden Kontrollpersonen. Um Unterschiede nachzuweisen, wurde ein multivariantes Mustererkennungsverfahren angewandt. Damit gelang es Klassifikatoren zu trainieren, die zwischen einem gesunden und einem kranken Kollektiv auf Einzelfallebene unterscheiden können. Dabei stießen die Forscher auf frappierende Unterschiede, aber auch auf Gemeinsamkeiten: Aus den MRT-Daten der Hochrisikoprobanden mit späterem Ausbruch einer Schizophrenie konnten die Münchener Psychiater Muster neuroanatomischer Veränderungen extrahieren, die diese Personen von Gesunden signifikant unterscheiden. Bereits vor Ausbruch der Schizophrenie konnten in morphometrischen Messungen Volumenabnahmen im fronto-tempo-parietalen Netzwerk nachgewiesen werden. Zugleich spiegelten die neuroanatomischen Unterschiede die klinische Entwicklung der Patienten mit hoher Genauigkeit wider - die Trefferquote lag zwischen $80 \%$ und $90 \%$. Bei Schizophrenie scheint sich also das Gehirn nicht in der gleichen Weise zu entwickeln wie das der gesunden Kontrollprobanden. „Das Gehirn der Patienten sah fünf bis sechs Jahre älter aus“, so Koutsouleris. Erstaunlicherweise wiesen Patienten mit unipolarer Depression ganz ähnliche strukturelle Veränderungen im Gehirn auf. Bei ihnen war der Abbau der Hirnsubstanz den gesunden Kontrollpersonen allerdings „nur“ um vier Jahre voraus. In Zukunft werde es nun darum gehen, nach spezifischen neuroanatomischen Unterschieden in den Gehirnen von Patienten mit Schizophrenie und Depression zu fahnden und die den beiden Krankheiten zugrunde liegenden pathogenetischen Mechanismen besser zu verstehen. Abdol A. Ameri, freier Medizinjournalist

Symposium „Brain imaging across mental disorders: Early detection and treatment", 21. EPA, Nizza, 6.-9.4.2013

\section{Der Rat vom Kollegen: Seien Sie gut zu sich selbst!}

\author{
Ob man es Burnout nennt, Anpassungsstörung oder einfach psychischen Erschöpfungszustand: Psychiater haben \\ ein erhöhtes Risiko, in einen solchen Zustand zu geraten. Die Folgen sind bekannt. Es ist also ratsam, sich beizeiten \\ nicht nur um die psychische Gesundheit seiner Patienten zu kümmern, sondern auch um die eigene.
}

So allgegenwärtig wie es in der Laienpresse ist, so umstritten ist es unter Psychiatern: Das Konzept „Burnout“. Professor Wulf Rössler, Psychiatrische Universitätsklinik Zürich, kann dem Medienrummel aber durchaus etwas abgewinnen: „Noch nie zuvor sind so viele Menschen zu mir gekommen und haben gesagt ,Ich habe eine psychische Krankheit'. Damit kann man dann arbeiten."
Burnout ist keine eigentliche ICD-10-Diagnose, kann aber unter dem Zusatzcode Z73 „Probleme mit Bezug auf Schwierigkeiten bei der Lebensbewältigung “ vermerkt werden. Die gängigsten ICD-Codes, die Psychiater im Zusammenhang mit Burnout verwenden, sind F43.2 - Anpassungsstörung und F48.0 - Neurasthenie. Als psychisches Syndrom wurde der Begriff Burnout erstmals 1975 von dem US-amerikanischen Psychoanalytiker 